\title{
NOUVELLE
}

\section{Expression ectopique du récepteur membranaire de l'interleukine 6 dans I'hypertension artérielle pulmonaire}

Ly Tu $^{1,2}$, Carole Phan ${ }^{1,2}$, Marc Humbert ${ }^{1-3}$, Christophe Guignabert ${ }^{1,2}$
${ }^{1}$ Inserm UMR_S 999, hôpital Marie Lannelongue,

133, avenue de la Résistance, 92350 Le Plessis-Robinson, France.

${ }^{2}$ Faculté de médecine, université Paris-Sud et université Paris-Saclay, Le Kremlin-Bicêtre, France.

${ }^{3} \mathrm{AP}-\mathrm{HP}$, service de pneumologie, centre de référence de I'hypertension pulmonaire sévère, DHU thorax innovation, Hôpital de Bicêtre, Le Kremlin-Bicêtre, France.

christophe.guignabert@inserm.fr

\section{L’hypertension artérielle pulmonaire}

Le terme «hypertension artérielle pulmonaire » (HTAP) regroupe un ensemble de maladies cardio-pulmonaires qui sont caractérisées par une augmentation de la pression artérielle pulmonaire moyenne et des résistances vasculaires pulmonaires, conduisant in fine à une insuffisance cardiaque droite et au décès. En France, la prévalence moyenne de I'HTAP est de 15 cas par million d'habitants avec une prédominance féminine. Bien que la prise en charge ait considérablement évolué ces dernières années, il n'existe malheureusement pas de traitement curatif de I'HTAP. Les thérapies actuelles restent essentiellement palliatives et ne ciblent pas directement les mécanismes physiopathologiques clés impliqués dans le remodelage du lit vasculaire pulmonaire, qui vont être responsables du déclin fonctionnel progressif des patients. La survie à 5 ans est inférieure à $60 \%$. La transplantation cardio-pulmonaire ou bi-pulmonaire est la seule solution chez les patients réfractaires au traitement médical avec un taux de survie de 52 à $75 \%$ à 5 ans.

Les progrès récents réalisés dans le déchiffrage de la physiopathologie de I'HTAP soulignent clairement que les processus de développement et d'entretien du remodelage de la circulation pulmonaire sont complexes et multifactoriels [1]. Parmi ces acteurs, les perturbations des fonctions de l'endothélium pulmonaire, l'accumulation excessive de cel- lules de la paroi vasculaire, tout comme l'infiltration périvasculaire de cellules inflammatoires jouent des rôles critiques [1]. Cependant, les mécanismes exacts sous-jacents sont encore largement méconnus. Au cours des deux dernières décennies, l'accumulation des connaissances autour du rôle de l'inflammation associée à I'HTAP s'est cependant accélérée [2] $(\rightarrow)$.

$(\rightarrow)$ Voir la Synthèse de F. Perros et al., $\mathrm{m} / \mathrm{s}$ $n^{\circ} 6-7$, juin-juillet Une forte augmen2013, page 607 tation de facteurs de l'inflammation, au niveau circulant et au niveau pulmonaire, est observée chez les patients HTAP, caractérisée notamment par des niveaux anormalement élevés de plusieurs cytokines et chimiokines pro-inflammatoires et la présence plus ou moins importante d'infiltrats inflammatoires transmuraux et périvasculaires. Quelques-uns de ces facteurs pro-inflammatoires peuvent être reliés à la sévérité de certaines formes d'HTAP $[3,4]$, notamment l'interleukine (IL)-6.

Sur-activation des voies de I'IL-6 dans I'HTAP humaine et expérimentale

L'IL-6 est une cytokine essentielle au système immunitaire qui possède de nombreuses fonctions allant de la régulation du cycle cellulaire à la modulation de l'état de différenciation des cellules inflammatoires. Or, l'IL-6 et sa voie de signalisation sont maintenant décrites comme étant parmi les acteurs de premier plan dans la pathogenèse de I'HTAP, en raison de leurs rôles critiques dans l'augmentation de la susceptibilité et de la progression du remodelage vasculaire pulmonaire. En effet, notre laboratoire a joué un rôle déterminant dans l'obtention d'éléments clés supportant un rôle de la voie de signalisation de I'IL-6/gp130' dans les changements structurels et fonctionnels du lit vasculaire pulmonaire associé à I'HTAP [5-8]. L'administration chronique d'IL-6 recombinante chez les rongeurs est en effet suffisante pour induire un remodelage vasculaire pulmonaire et augmenter la susceptibilité de ces animaux au développement de la maladie dans les modèles expérimentaux d'étude de I'HTAP [9]. La surexpression d'IL-6 est également suffisante pour induire un remodelage vasculaire pulmonaire et une hypertension pulmonaire spontanée chez la souris, tandis que la déficience en IL-6 a un effet protecteur contre le développement d'hypertension pulmonaire induite par l'hypoxie chronique $[7,10]$. Nous avons récemment pu mettre en évidence que la production excessive d'IL-6 par l'endothélium pulmonaire dysfonctionnel contribuait à une couverture péricytaire excessive des artères pulmonaires remodelées dans I'HTAP expérimentale et humaine, facilitant leur muscularisation $[6,11]$. Bien que ces différents éléments soulignent un lien entre la signalisation

\footnotetext{
${ }^{1}$ La glycoprotéine gp130 est une glycoprotéine transmembranaire qui s'associe au récepteur de l'IL-6 afin de transmettre le signal induit par la liaison de la cytokine.
} 


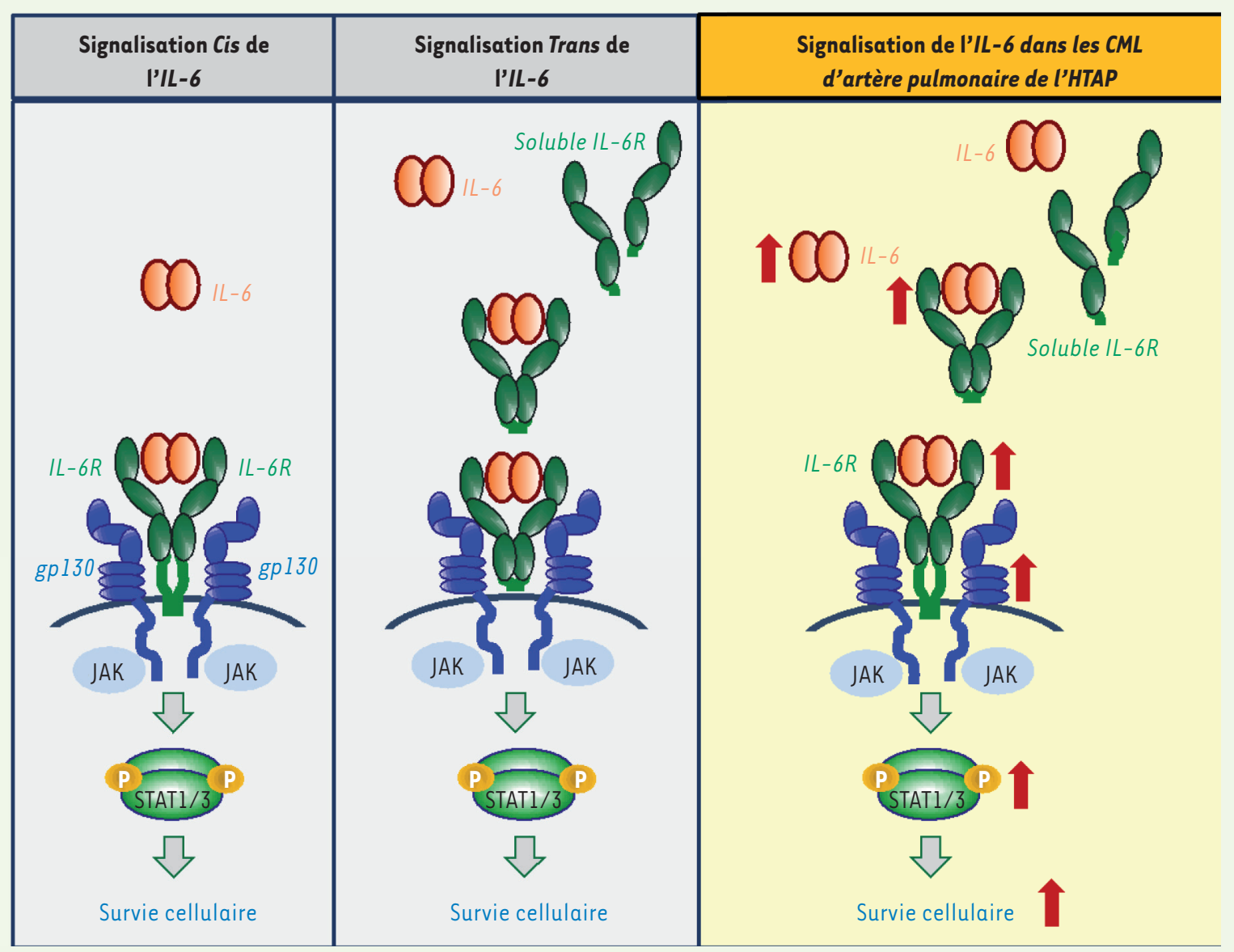

Figure 1. Dérégulation des voies de l'IL-6 chez les patients avec hypertension artérielle pulmonaire. Les deux voies de signalisation de l'interleukine-6 (IL-6) : voie cis (panneau de gauche) et voie trans (au centre). Représentation schématique de la signalisation dérégulée des voies de l'IL-6 dans les cellules musculaires lisses d'artère pulmonaire (CML-AP) de patients avec hypertension artérielle pulmonaire (HTAP, panneau de droite). IL-6R : récepteur de l'IL-6, gp130 : glycoprotéine membranaire ; JAK : janus kinase ; STAT : signal transducers and activators of transcription.

cellulaire induite par I'IL-6 et la progression de l'HTAP, les mécanismes sousjacents unissant ces observations restaient jusqu'à présent méconnus.

Deux voies de signalisation différentes ont été décrites pour l'IL-6 : la voie cis (également dite voie classique) et la voie trans (Figure 1). Dans la voie classique, I'IL-6 se lie à la surface membranaire par l'intermédiaire de son récepteur, l'IL-6R, et conduit à sa dimérisation avec la protéine gp130 qui va initier la transduction du signal intracellulaire aboutissant à l'activation de la voie de signalisation transduite par JAK/STAT (janus kinase/ signal transducers and activators of transcription). En situation physiolo- gique, cette voie classique est limitée aux hépatocytes, aux cellules épithéliales et intestinales, aux mégacaryocytes, aux polynucléaires neutrophiles, aux monocytes/macrophages et aux lymphocytes $\mathrm{CD}^{+}$ainsi qu'aux plasmocytes puisque l'expression membranaire de l'IL-6R est restreinte à ces différents types cellulaires. Les cellules qui n'expriment pas I'IL-6R membranaire peuvent néanmoins répondre à I'IL-6 au travers de la voie de trans-signalisation. Dans cette voie, I'IL-6 se lie à la forme soluble de l'IL-6R (ou sIL-6R), ce complexe IL-6/sIL-6R peut ensuite se lier à la glycoprotéine gp130 membranaire qui est présente de manière ubiquitaire sur toutes les cel- lules. La voie de trans-signalisation permet donc d'élargir le spectre d'action de I'IL-6, puisque les cellules n'exprimant pas I'IL-6R à leur surface vont pouvoir être stimulées par le complexe |L-6/s|L6R. II est maintenant reconnu que certains aspects des désordres inflammatoires et néoplasiques sont transduits par cette voie de trans-signalisation de I'IL-6.

C'est en suivant l'expression des différentes protéines constitutives des voies cis et trans que nous avons pu montrer que ces deux voies étaient également impliquées dans I'HTAP humaine et expérimentale [8]. En plus de la surabondance d'IL-6 et de sIL-6R dans le 
sérum des patients, nous avons noté une surexpression de la gp 130 et une expression ectopique de I'IL-6R à la surface des cellules musculaires lisses d'artère pulmonaire (CML-AP) de patients avec HTAP idiopathique (HTAPi). Des analyses fonctionnelles réalisées in vitro, menées avec des CML-AP de patients HTAPi ou de sujets contrôles, ont permis de démontrer que l'activation de l'IL-6R dans les CML-AP HTAPi induit l'expression de facteurs anti-apoptotiques, comme MCL-1 (myeloid cell leukemia-1) et $\mathrm{Bcl}-2$ (B-cell lymphoma-2), facilitant ainsi la survie de ces cellules dans la paroi des artères pulmonaires remodelées. La mise en place de cette signalisation pathologique de I'IL-6R dans les CML-AP a pu être validée dans deux modèles précliniques d'étude de I'HTAP : le modèle monocrotaline ${ }^{2}$ (MCT) et le modèle d'administration de SU5416 combiné à l'hypoxie chronique $(\mathrm{SuHx})^{3}$. En cohérence avec ces données, l'invalidation spécifique du gène codant l'IL-6R dans le muscle lisse chez la souris (souris Sm22-Cre/ll6rflox/flox) confère une moindre susceptibilité au développement d'hypertension pulmonaire induite par l'exposition chronique à l'hypoxie [8]. Nous avons également pu mettre en évidence des effets bénéfiques d'un traitement chronique par un antagoniste spécifique de I'IL-6R (le 20S, 21-epoxyresibufogenin-3-formate ou $\varepsilon R B F$ ) sur le remodelage des artères pulmonaires et l'infiltration pulmonaire de cellules

\footnotetext{
${ }^{2}$ La monocrotaline est un alcaloïde de la famille de la pyrrolizidine qui se trouve dans les tiges, feuilles et graines de Crotalaria spectabilis. La toxicité de la monocrotaline est essentiellement hépatique et cardio-pulmonaire. L'hypertension pulmonaire à la monocrotaline ressemble à l'HTAP par sa gravité hémodynamique et histopathologique et sa mortalité naturelle particulièrement élevée.

${ }^{3}$ Le modèle sugen/hypoxie (SuHx) est un modèle sévère d'hypertension pulmonaire principalement étudié chez le rat. I est issu d'une amélioration du modèle d'hypoxie chronique qui consiste à maintenir les animaux en basse concentration d'oxygène $(10 \%)$ pour une période de 3 semaines. Ce modèle SuHx combine l'injection d'un antagoniste du récepteur 2 du facteur de croissance de l'endothélium vasculaire (VEGFR2) par le composé SU5416 (également connu sous le nom de sugen ou semaxanib) à une hypoxie de trois semaines puis à un retour en conditions normoxiques pour une durée de 5 semaines. C modèle SuHx se caractérise non seulement par sa sévérité, mais aussi par la formation progressive de lésions vasculaires pul monaires occlusives similaires à celles retrouvées dans l'HTAP.
}

inflammatoires dans les deux modèles précliniques $\mathrm{MCT}^{2}$ et $\mathrm{SuHx}^{3}$. Ces effets étaient associés à la réduction de l'activation des kinases JAK/STAT [8]. Ainsi, cette découverte jette les bases de thérapies ciblant la signalisation de l'IL-6R pour les patients atteints d'HTAP.

\section{Conclusions et perspectives thérapeutiques}

En conclusion, de nombreuses données indiquent que l'inflammation est une des voies physiopathologiques du développement de l'HTAP et que l'expression ectopique de l'IL-6R à la surface des CML-AP pourrait représenter une cible pertinente pour lutter contre sa progression. II existe déjà plusieurs stratégies d'inhibition des voies de l'IL-6 qui ciblent: (1) soit directement I'IL-6; (2) soit son récepteur (IL-6R) affectant les voies cis et trans ; (3) soit le complexe IL-6/sIL-6R par l'utilisation de protéines de fusion gpl30 inhibant sélectivement la signalisation trans; (4) soit, enfin, la transduction intracellulaire du signal en faisant appel à des inhibiteurs ciblant notamment la phosphorylation de JAK ou de STAT. Les travaux menés par Hashimoto-Kataoka et ses collaborateurs ont également montré que le blocage de l'IL-21, qui peut être induite par l'IL-6, peut également permettre de limiter certaines actions des voies de I'IL-6 et notamment celles liées à l'activation macrophagique dans I'HTAP expérimentale [12]. L'efficacité de traitements chroniques avec des protéines de fusion gp130 a aussi été démontrée dans les modèles expérimentaux d'hypertension pulmonaire [13]. Cependant cette dernière stratégie ainsi que celle utilisant des inhibiteurs de JAK ou STAT ne sont pas spécifiques des voies de I'IL-6. La gpl30 est en effet un corécepteur impliqué dans les voies de signalisation induites par l'IL11, I'IL-27, du leukemia inhibitory factor (LIF) et celles de l'oncostatine M (OSM). Ces stratégies peuvent donc potentiellement impacter d'autres mécanismes de l'inflammation et de l'immunité.

Bien que les capacités de pléïotropisme, de redondance d'actions et d'intercon- nections des systèmes d'activation ainsi que les potentiels effets indésirables soient de clairs obstacles à franchir, le développement de stratégies anti-inflammatoires dans I'HTAP reste très prometteur. Récemment, une étude ouverte de phase 2 pour évaluer la tolérance et l'efficacité du tocilizumab chez les patients atteints d'HTAP a été initiée (ClinicalTrials, gov NCT02676947). Le tocilizumab est un anticorps monoclonal humanisé qui reconnaît I'IL-6R et bloque à la fois les signalisations cis et trans de l'IL-6. II est actuellement approuvé pour le traitement de la polyarthrite rhumatoïde. $\diamond$

IL6R is the key therapeutical target in pulmonary arterial hypertension

\section{REMERCIEMENTS}

Ce travail a notamment reçu le support de l'ANR (ANR-16-CE17-0014 - TAMIRAH), du département hospitalo-universitaire (DHU) thorax innovation (TORINO) et de la chancellerie des universités de Paris. Carole Phan a reçu une allocation doctorale $d u$ fonds de recherche en santé respiratoire/fondation du souffle (FRSR/FdS).

\section{LIENS D'INTÉRÊT}

Les auteurs déclarent n'avoir aucun lien d'intérêt concernant les données publiées dans cet article.

\section{RÉFÉRENCES}

1. Guignabert C, Tu L, Girerd B, et al. New molecular targets of pulmonary vascular remodeling in pulmonary arterial hypertension: importance of endothelial communication. Chest 2015 ; 147 : 529-37.

2. Perros F, Humbert M, Cohen-Kaminski S. Hypertension pulmonaire artérielle : un parfum d'auto-immunité. Med Sci (Paris) 2013 ; 29: 607-16.

3. Cracowski JL, Chabot F, Labarere J, et al. Proinflammatory cytokine levels are linked to death in pulmonary arterial hypertension. Eur Respir J 2014 ; 43 : 915-7.

4. Soon E, Holmes AM, Treacy CM, et al. Elevated levels of inflammatory cytokines predict survival in idiopathic and familial pulmonary arterial hypertension. Circulation 2010; $122: 920-7$

5. Humbert M, Monti G, Brenot F, et al. Increased interleukin- 1 and interleukin- 6 serum concentrations in severe primary pulmonary hypertension. Am J Respir Crit Care Med 1995 ; 151 : 1628-31.

6. Ricard N, Tu L, Le Hiress M, et al. Increased pericyte coverage mediated by endothelial-derived fibroblast growth factor- 2 and interleukin- 6 is a source of smooth muscle-like cells in pulmonary hypertension. Circulation 2014 ; 129 : 1586-97.

7. Savale L, Tu L, Rideau D, et al. Impact of interleukin-6 on hypoxia-induced pulmonary hypertension and lung inflammation in mice. Respir Res $2009 ; 10: 6$.

8. Tamura Y, Phan C, Tu L, et al. Ectopic upregulation of membrane-bound IL6R drives vascular remodeling in pulmonary arterial hypertension. J Clin Invest 2018 ; $128: 1956-70$. 


\section{RÉFÉRENCES}

9. Golembeski SM, West J, Tada Y, Fagan KA. Interleukin-6 causes mild pulmonary hypertension and augments hypoxia-induced pulmonary hypertension in mice. Chest 2005 ; 128 : S572-3.

10. Steiner MK, Syrkina OL, Kolliputi N, et al. Interleukin-6 overexpression induces pulmonary hypertension. Circ Res 2009 ; 104 : 236-44, 28p following 44.
11. Le Hiress M, Tu L, Ricard N, et al. Proinflammatory signature of the dysfunctional endothelium in pulmonary hypertension. Role of the macrophage migration inhibitory factor/CD74 complex. Am J Respir Crit Care Med 2015 ; 192 : 983-97.

12. Hashimoto-Kataoka T, Hosen $\mathrm{N}$, Sonobe $\mathrm{T}$, et al. Interleukin-6/interleukin-21 signaling axis is critical in the pathogenesis of pulmonary arterial hypertension. Proc Natl Acad Sci USA 2015 ; 112 : ع2677-86.

\title{
NOUVELLE
}

\section{Retournement de situation dans la dissémination des cancers colorectaux}

\author{
Olivier Zajac ${ }^{1}$, Fanny Jaulin²
}

> Les métastases, tumeurs secondaires formées à distance, sont responsables de $90 \%$ des décès de patients atteints de cancer. La compréhension des mécanismes de dissémination des cellules tumorales est donc une priorité si nous voulons interférer avec cette cascade métastatique.

Les carcinomes sont des cancers formés à partir de tissus épithéliaux. Ils représentent environ $85 \%$ des tumeurs recensées. Les épithéliums, à l'interface entre les organes et le milieu extérieur, assurent la protection et l'homéostasie des individus. Ils sont constitués de cellules épithéliales étroitement cohésives et hautement polarisées. Les jonctions intercellulaires adhérentes, organisées autour de la $\varepsilon$-cadhérine, et jonctions serrées, organisées autour des claudines, contrôlent la perméabilité de la monocouche épithéliale. Chaque cellule est polarisée, son pôle apical faisant face à la cavité luminale de l'organe alors que le pôle basolatéral est au contact du milieu interne, notamment de la lame basale (Figure 1). Les carcinomes se développent à partir d'une cellule unique qui prolifère initialement de façon localisée, constituant un cancer in situ. Lorsque la maladie progresse, les cellules tumorales quittent le tissu épithé-

lial pour envahir le microenvironnement péritumoral principalement constitué de collagène-l et de fibroblastes. Elles $y$ atteindront différentes voies de dissémination, comme les vaisseaux lymphatiques et sanguins, ou encore certaines cavités qu'elles emprunteront pour accéder à des organes secondaires.

La locomotion des cellules tumorales dans leur microenvironnement est une étape cruciale de la cascade métastatique et une cible thérapeutique attrayante. Les mécanismes d'invasion des cellules tumorales ont fait l'objet de nombreuses études, dont la majorité portent sur la transition épithélio-mésenchymateuse (EMT) [1] $(\rightarrow)$.

\section{$\rightarrow$ Voir la Synthèse de C. Moyret-Lalle et al., $\mathrm{m} / \mathrm{s} \mathrm{n}^{\circ} 8-9$, août-septembre 2016, page 725}

Ce programme transcriptionnel module certaines étapes du développement embryonnaire comme la gastrulation et la formation du tube neural [2]. L'EMT peut être activée par différentes voies de signalisation, notamment celle du TGF- $\beta$ (transforming growth factor- $\beta$ ). Ce processus peut participer à la dissémination des carcinomes en permettant l'individualisation des cellules épithéliales transformées grâce à la répression de l'expression du gène codant l' $\varepsilon$-cadhérine, entraînant la dissolution des jonc-
13. Jasiewicz M, Knapp M, Waszkiewicz $\varepsilon$, et al. Enhanced IL- 6 trans-signaling in pulmonary arterial hypertension and its potential role in disease-related systemic damage. Cytokine $2015 ; 76$ : 187-92.

\author{
${ }^{1}$ Institut Curie, 26, rue d'UIm 75005 Paris, \\ France. \\ ${ }^{2}$ Institut Gustave Roussy, \\ 114 , rue Édouard Vailland, B2M, \\ 94800 Villejuif, France. \\ fanny.jaulin@gustaveroussy.fr
}

tions intercellulaires. Les cellules perdent alors leur architecture épithéliale, leur polarité apico-basolatérale, et gagnent des capacités migratoires et protéolytiques leur permettant d'envahir les tissus sains du patient [2]. En observant des explants de tumeurs ex-vivo, Peter Friedl avait montré, dès 1995, la capacité des cellules tumorales à envahir collectivement les tissus [3]. Non spécifique des cancers, la migration collective orchestre de nombreuses étapes du développement embryonnaire. Elle est caractérisée par une coopération entre les cellules de la cohorte migrant grâce au maintien de leurs jonctions intercellulaires. Récemment, l'utilisation des modèles murins de cancers du sein a permis de démontrer que des cohortes de cellules tumorales atteignent la circulation hématogène et initient la formation de métastases [4-6]. La contribution respective des cellules isolées et des cohortes à la formation de métastases est actuellement très débattue $[7,8]$. La nature du modèle expérimental choisi semble fortement influencer le mode d'invasion emprunté par les cellules cancéreuses, mais l'acquisition des propriétés invasives semble toujours associée à une perte de l'architecture épithéliale [9]. Au-delà des modèles, les mécanismes entraînant la dissémination 